\title{
Protein tyrosine kinase 7 plays a tumor suppressor role by inhibiting ERK and AKT phosphorylation in lung cancer
}

\author{
JI-HEE KIM ${ }^{1}$, JUNHYE KWON ${ }^{1,2}$, HAE WON LEE $^{3}$, MOON CHUL KANG $^{3}$, \\ HYEON-JOON YOON ${ }^{1}$, SEUNG-TAEK LEE ${ }^{4}$ and JONG HO PARK ${ }^{1,3}$
}

\author{
${ }^{1}$ Department of Translational Research, Korea Cancer Center Hospital, Korea Institute of Radiological and \\ Medical Sciences, Seoul 139-706; ${ }^{2}$ Research Institute of Women's Health, Sookmyung Women's University, \\ Seoul 140-742; ${ }^{3}$ Department of Thoracic Surgery, Korea Cancer Center Hospital, Korea Institute of \\ Radiological and Medical Sciences, Seoul 139-706; ${ }^{4}$ Department of Biochemistry, College of \\ Life Science and Biotechnology, Yonsei University, Seoul 120-749, Republic of Korea
}

Received January 29, 2014; Accepted April 9, 2014

DOI: $10.3892 /$ or.2014.3164

\begin{abstract}
Protein tyrosine kinase 7 (PTK7) is a catalytically inactive receptor tyrosine kinase that is also known as colon carcinoma kinase-4 (CCK-4). Recent reports have shown that PTK7 plays an important role in carcinogenesis, and it is known to be upregulated in gastric, colon and esophageal cancer, as well as in liposarcoma. However, the role of PTK7 in lung cancer has not been investigated. The aim of the present study was to investigate the expression levels and the role of PTK7 in lung cancer. We found that PTK7 expression was downregulated at the mRNA as well as protein levels in human lung squamous cell carcinoma (LSCC). Upon investigation of the functional role of PTK7 in LSCC, we found that overexpression of PTK7 in LSCC cells resulted in inhibition of cell proliferation, invasion and migration. Furthermore, we confirmed that these phenotypic changes are associated with the inactivation of AKT and ERK. Our findings suggest that PTK7 has different oncogenic roles in organs and target tumors.
\end{abstract}

\section{Introduction}

Lung cancer is the leading cause of cancer-related mortality worldwide (1). The prognosis of lung cancer patients is poor despite the development of several new therapeutic modalities, and this may be attributed to the difficulties associated with the detection of lung cancer in its early stages. Therefore, new approaches and alternative diagnostic modalities are urgently needed.

Correspondence to: Dr Jong Ho Park, Department of Thoracic Surgery, Korea Cancer Center Hospital, Korea Institute of Radiological and Medical Sciences, 75 Nowon-Ro, Nowon-Ku, Seoul 139-706, Republic of Korea

E-mail: jhpark@kcch.re.kr

Key words: lung squamous cell carcinoma, protein tyrosine kinase 7
Squamous cell carcinoma (SCC) of the lung, a major type of non-small cell lung carcinoma (NSCLC), is more strongly linked with smoking than other forms of $\operatorname{NSCLC}(2,3)$. The genes responsible for lung SCC (LSCC) have been identified, but the molecular origins of LSCC remain unclear. In our studies on the mechanism of tumorigenesis in LSCC, we focused on protein tyrosine kinase 7 (PTK7), which has been previously reported to play a key role in carcinogenesis (4-8).

PTK7, also known as colon carcinoma kinase-4 (CCK-4), is a catalytically inactive receptor tyrosine kinase, cloned from colon carcinoma tissue (4). It is composed of a transmembrane region, seven immunoglobulin domains, and a C-terminal domain that shows homology to tyrosine kinases.

PTK7 expression has been shown to be high in several tumors, including gastric, colon, esophageal cancer, and liposarcoma (4-7). PTK7 knockdown is known to inhibit proliferation of liposarcoma cells, esophageal cancer cells, and colon cancer cells, and silencing of PTK7 in colon cancer cells induces caspase-10-dependent apoptosis via the mitochondrial pathway (6-8). These studies suggested the probability of an oncogenic role of PTK7 in gastric, colon and esophageal cancer, as well as in liposarcoma. However, PTK7 has been reported to be downregulated in clear cell renal cell carcinoma, metastatic melanoma and breast cancer cell lines (9-11).

Taken together, these previous findings suggested that PTK7 could exert dual effects (upregulation or downregulation), depending on specific tissues or tumors, although the exact mechanism is unknown. In this study, we attempted to explore the role of PTK7 in lung cancer.

\section{Materials and methods}

Recruitment of patients and preparation of tissue samples. This study was approved by the institutional review board of the Korea Cancer Center Hospital. For RT-PCR and western blot analysis, frozen samples of tumor and adjacent normal lung tissue were obtained from 12 lung cancer patients who underwent curative surgery for LSCC at the Korea Cancer Center Hospital. Pathologic staging was performed based on 
the recommendations in the $7^{\text {th }}$ edition of the report of the American Joint Committee on Cancer.

Cell lines. Human LSCC cell lines, HCC-1588 and HCC-95, were purchased from the Korean Cell Line Bank (http://cellbank.snu.ac.kr); SW900, SK-MES-1, Calu-1 and the normal human lung fibroblast cell line WI-38 were purchased from the American Type Culture Collection. HCC-1588 and HCC-95 were maintained in RPMI-1640 medium supplemented with $10 \%$ fetal bovine serum (FBS) and $100 \mathrm{U} / \mathrm{ml}$ penicillin and streptomycin. SK-MES-1 and WI-38 cells were maintained in EMEM medium supplemented with $10 \%$ FBS and $100 \mathrm{U} / \mathrm{ml}$ penicillin and streptomycin. SW900 and Calu-1 cells were maintained in L-15 and McCoy's 5A media supplemented with $10 \% \mathrm{FBS}$ and $100 \mathrm{U} / \mathrm{ml}$ penicillin and streptomycin, respectively. All cells were cultured at $37^{\circ} \mathrm{C}$ in the presence of $5 \%$ $\mathrm{CO}_{2}$.

Transfection. Cells were transfected with $2 \mu \mathrm{g}$ of pcDNA3 (Invitrogen, Carlsbad, CA, USA) and pcDNA3-PTK7Flag (12) by using FuGENE HD reagents (Promega, Madison, WI, USA) according to the manufacturer's instructions.

RNA isolation and RT-PCR. Total RNA was isolated from frozen lung samples (SCC and normal lung tissues) and cell lines using an RNeasy Mini Kit (Qiagen, Cambridge, MA, USA) according to the manufacturer's protocol. Single-stranded cDNA was produced by reverse-transcription of total RNA $(2 \mu \mathrm{g})$ by using an oligo-dT primer and Superscript II reverse transcriptase (Invitrogen). RT-PCR was performed (13) with the following sets of synthesized primers: PTK7 forward, 5'-AGAGATGCCCCATGGTGGGC-3' and reverse, 5'-ACGG CTTGCTGTCCACGGTG-3'. GAPDH-specific primers were used as internal controls.

Cell proliferation assay. Cell growth was determined by the MTS assay using the CellTiter $96{ }^{\circledR}$ Aqueous One Solution Cell Proliferation Assay kit (Promega). Cells (2x10\%3ell) were seeded in 96-well plates after transfection. After incubation for 24, 48, 72 and $96 \mathrm{~h}, 20 \mu \mathrm{l}$ of MTS solution was added to each well. Plates were incubated for an additional $1-2 \mathrm{~h}$ at $37^{\circ} \mathrm{C}$ in a $5 \% \mathrm{CO}_{2}$ environment, and then the absorbance at $490 \mathrm{~nm}$ was recorded using a microplate reader (Biotek, Winooski, VT, USA) to calculate the percentage of surviving cells.

Migration and invasion assay. Cells (5x10\%/well) were seeded in a 24-well plate and incubated with fresh medium. After overnight incubation, a wound was introduced by scraping the monolayer with a $0.2-\mathrm{ml}$ micropipette tip. The attached cells were washed twice with PBS to remove debris, incubated for $48 \mathrm{~h}$ in RPMI-1640 supplemented with $10 \%$ FBS, and observed by light microscopy. The invasion assay was performed using BioCot $^{\mathrm{TM}}$ Matrigel $^{\mathrm{TM}}$ invasion chamber (8 $\mu \mathrm{m}, 24$-well; BD Biosciences, Franklin Lakes, NJ, USA). Cells were starved in serum-free medium overnight, trypsinized. Then, cells were suspended in FBS-free RPMI-1640 ( $2 \times 10^{3}$ cells) and were seeded into the upper compartment of the chamber, and $600 \mu \mathrm{l}$ of RPMI-1640 containing $10 \%$ FBS was placed in the lower chamber. After incubation for $24 \mathrm{~h}$ or $48 \mathrm{~h}$ at $37^{\circ} \mathrm{C}$ in a $5 \% \mathrm{CO}_{2}$, the cells remaining in the upper compartment of the chamber were removed. Cells on the lower surface of the chamber were fixed using $4 \%$ paraformaldehyde and stained with $0.5 \%$ crystal violet.

Western blot analysis. Proteins from frozen tissues and cell lines were extracted using RIPA buffer (Thermo Fisher Scientific, Waltham, MA, USA) containing protease inhibitors (Roche, Basel, Switzerland). Lysates containing the same amounts of protein were separated on $10 \%$ SDS-polyacrylamide gels and transferred onto nitrocellulose or PVDF membranes. Western blotting was performed as previously described (14) using the following antibodies: anti-phospho-ERK, anti-ERK, and anti- $\beta$-actin (Santa Cruz Biotechnology, Santa Cruz, CA, USA); anti-phospho-AKT (Ser473), and anti-AKT (Cell Signaling Technology, Beverly, MA, USA) primary antibodies and horseradish peroxidase-conjugated secondary antibodies (Santa Cruz Biotechnology). Rabbit anti-PTK7 anti-serum, which recognizes both human and murine PTK7, was used as previously described (13). The immune reactions were visualized using Immobilon Western Chemiluminescent HRP Substrate (Millipore, Bedford, MA, USA) and a LAS-3000 imaging system (Fuji, Tokyo, Japan).

Statistical analysis. Each experiment was performed at least three times. All values are reported as mean \pm SD. Differences between experimental groups and controls were assessed by Tukey's test using SigmaStat (SPSS, Inc., Chicago, IL, USA). The level of statistical significance was set at $\mathrm{P}<0.05$.

\section{Results}

Low expression of PTK7 in LSCC patient samples and LSCC cell lines. The expression of PTK7 in LSCC tissues was low at the mRNA level in 11/12 samples and at the protein level in 8/10 samples (Fig. 1). The LSCC cell lines also showed a significantly lower expression of PTK7 than the normal lung cell line WI-38 (Fig. 2). These results showed that PTK7 is downregulated in both LSCC tissues and LSCC cell lines.

Overexpression of PTK7 in transfected LSCC cells inhibits cell proliferation, wound healing and invasion. To confirm the biological role of PTK7 in LSCC, we transfected the LSCC cell lines HCC-95 and HCC-1588 with a PTK7-expression vector pcDNA3-PTK7-flag and with an empty vector pcDNA3. The results of the western blot analysis of the cell lines transfected with the PTK7-expression vector showed overexpression of PTK7 (Fig. 3A). The effect of this PTK7 overexpression on the proliferation of LSCC cells was evaluated by the MTS assay. The results of the MTS assay showed that the proliferation of cells transfected with the PTK7-expression vector was significantly less than that of cells transfected with the empty vector $(\mathrm{P}<0.05$; Fig. 3B). We then investigated the role of PTK7 in wound healing and invasion of HCC-95 and HCC-1588 cells. In the wound healing assay, control cells repaired the wound in $48 \mathrm{~h}$, whereas the PTK7-overexpressing cells showed significantly delayed wound repair (Fig. 3C). The invasive potential of the PTK7-overexpressing HCC-95 and HCC-1588 cells was $73 \%$ lower than that of control cells (Fig. 3D). The effect of PTK7 overexpression on LSCC cell growth was also assessed by the colony formation assay; however, both control and 
A

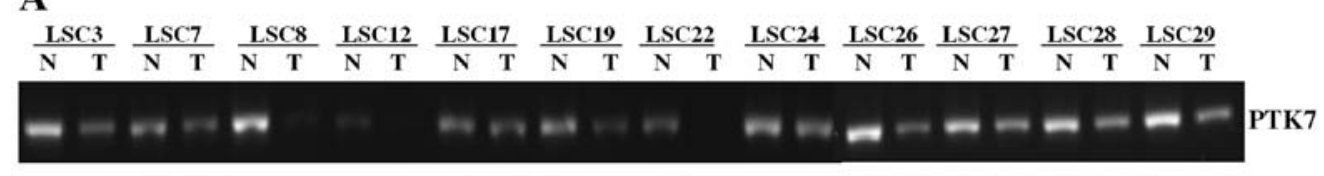

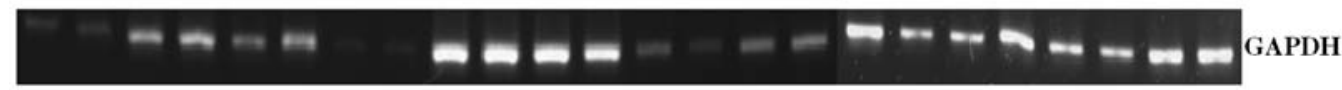

B

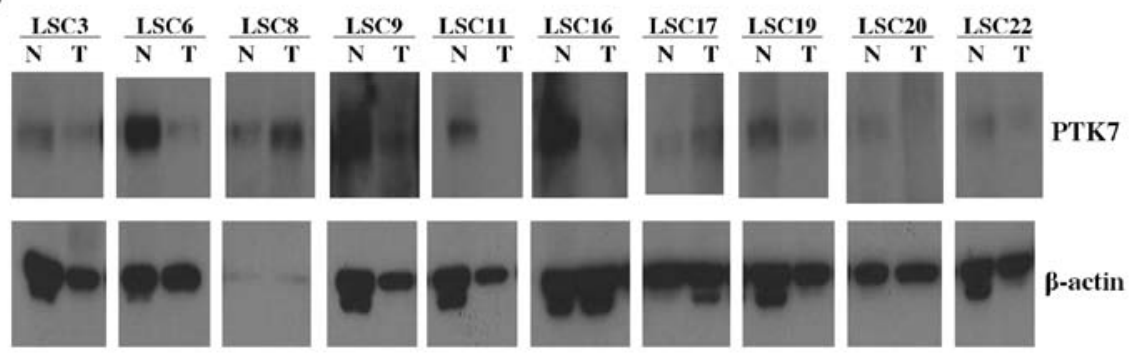

Figure 1. Downregulation of PTK7 in tumor samples from 10 LSCC patients. (A) Levels of PTK7 mRNA as determined by RT-PCR. GAPDH mRNA levels are shown for normalization. (B) PTK7 protein expression in LSCC tumor and non-tumor tissues as detected by western blotting. $\beta$-actin was used as the loading control. N, normal lung tissue, adjacent to the tumor; T, tumor tissue sample.

A

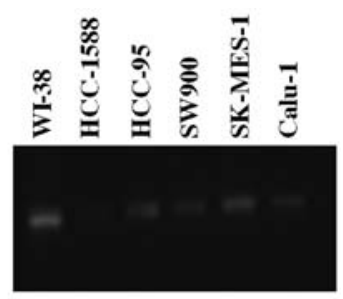

GAPDH

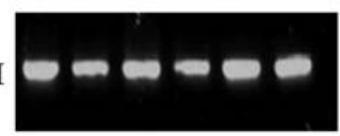

B

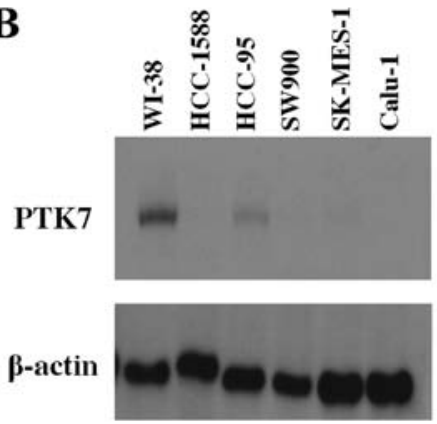

Figure 2. Downregulation of PTK7 in LSCC cell lines. (A) Expression of PTK7 at the mRNA level in LSCC cell lines and normal lung cell line as determined by RT-PCR. GAPDH mRNA levels are shown for normalization. (B) PTK7 protein expression in LSCC cell lines and normal lung cell line as detected by western blotting. $\beta$-actin was used as the loading control.

PTK7-overexpressing cells showed similar growth rates (data not shown).

Overexpression of PTK7 inhibits the activation of ERK and $A K T$ in LSCC cells. We conducted a microarray analysis to investigate genetic alterations in PTK7-overexpressed LSCC cell lines. Results of this analysis revealed mutations in the genes coding for AKT and ERK, p38, and JNK-enzymes involved in the mitogen activated protein kinase (MAPK) pathways (data not shown). This finding led us to consider that these pathways likely act together to regulate PTK7-influenced cell proliferation, invasion and migration. Therefore, we investigated the activation of AKT and MAPKs in PTK7overexpressed LSCC cells. In both HCC-95 and HCC-1588 cells, PTK7 overexpression decreased the phosphorylation levels of AKT and ERK (Fig. 4). However, activation of JNK and p38 were found to be similar in control as well as in PTK7 overexpressing cells (data not shown). These results suggest that PTK7 effectively modulates signaling pathways involving AKT and ERK, which are important determinants of cell proliferation, migration and invasion in LSCC cells.

\section{Discussion}

Several studies have reported the increase in PTK7 expression in gastric cancer, colon cancer, liposarcoma and esophageal cancer (4-7). We have also previously reported a correlation between high expression of PTK7 and poor prognosis in esophageal cancer and that knockdown of PTK7 in esophageal cancer cells inhibits cell proliferation, survival, invasion and migration through inhibition of AKT, ERK, FAK, JNK, and p38 MAPK (6). In addition, suppression of PTK7 has been shown to inhibit cell proliferation and to induce apoptosis via the mitochondria pathway in colon cancer cells (8). These results suggest that PTK could be regarded as an oncogene (5-8).

On the other hand, PTK7 has been shown to be downregulated in several types of cancer, including clear cell renal cell carcinoma, metastatic melanoma and pulmonary adenocarcinoma $(9,10,15)$. Easty et al $(10)$ explored the probable correlation between loss of PTK7 expression in melanoma cells and tumorigenicity. In addition, Endoh et al (15) and Garber et al (16) reported that decreased expression of PTK7 is correlated with poor clinical outcome. These findings suggested that PTK7 expression may be upregulated or down- 
A

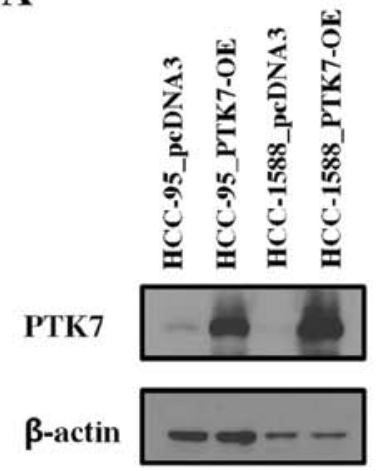

B

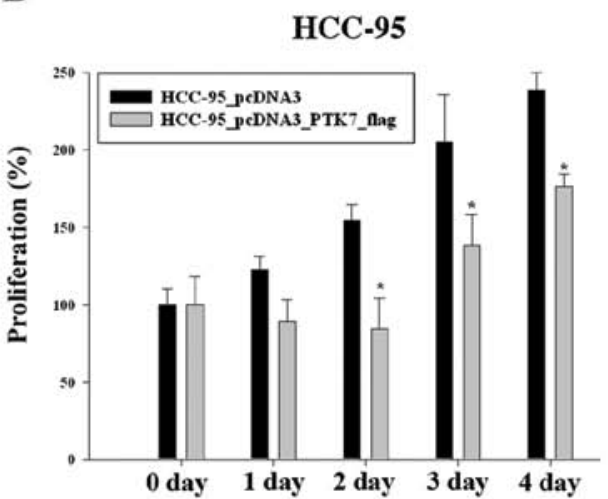

C
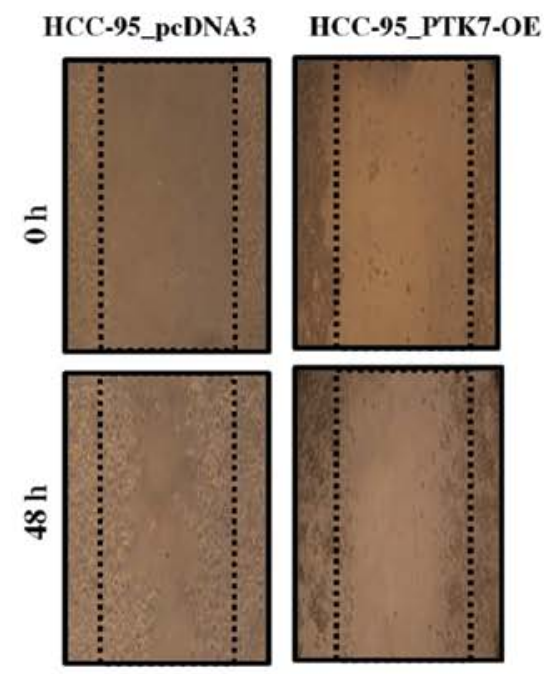

HCC-1588_peDNA3 HCC-1588_PTK7-UE
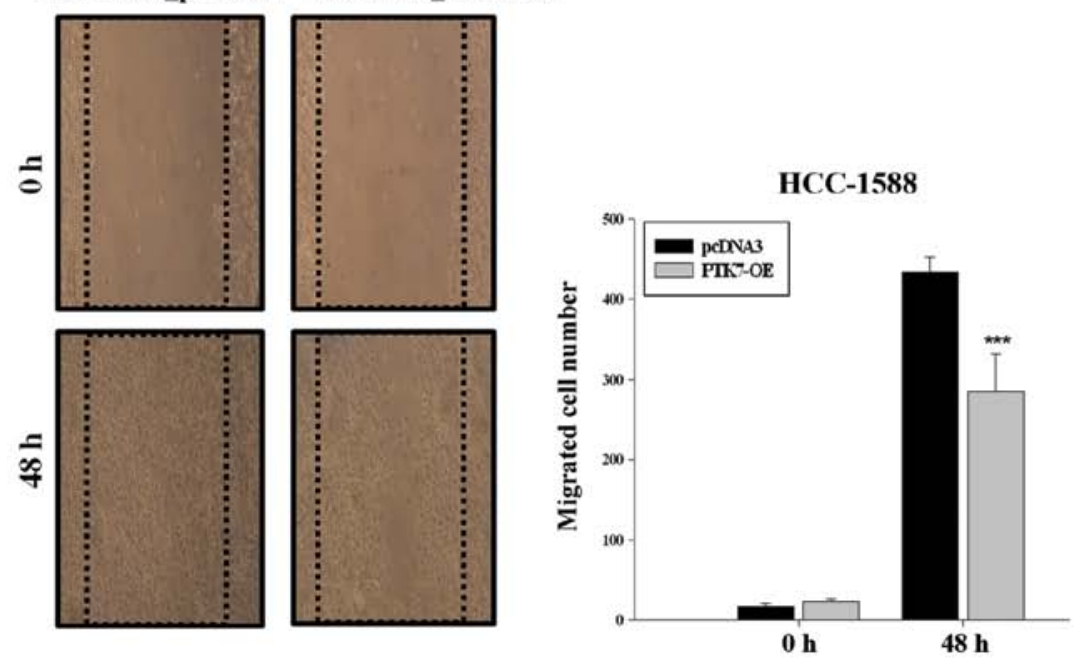

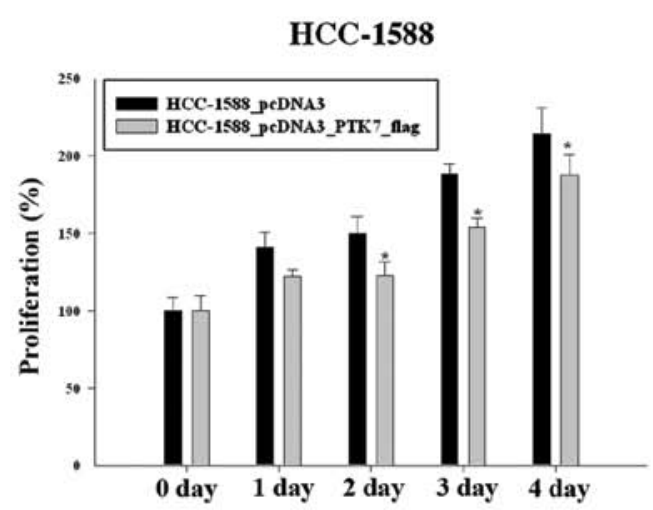

D
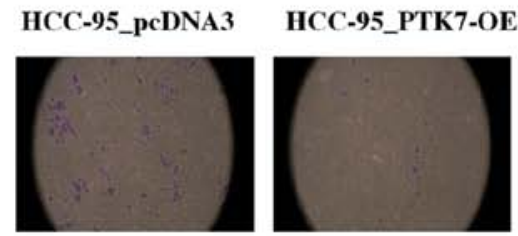

HCC-1588_pcDNA3 HCC-1588_PTK7-OE

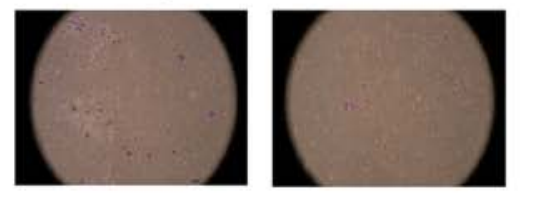

HCC-95
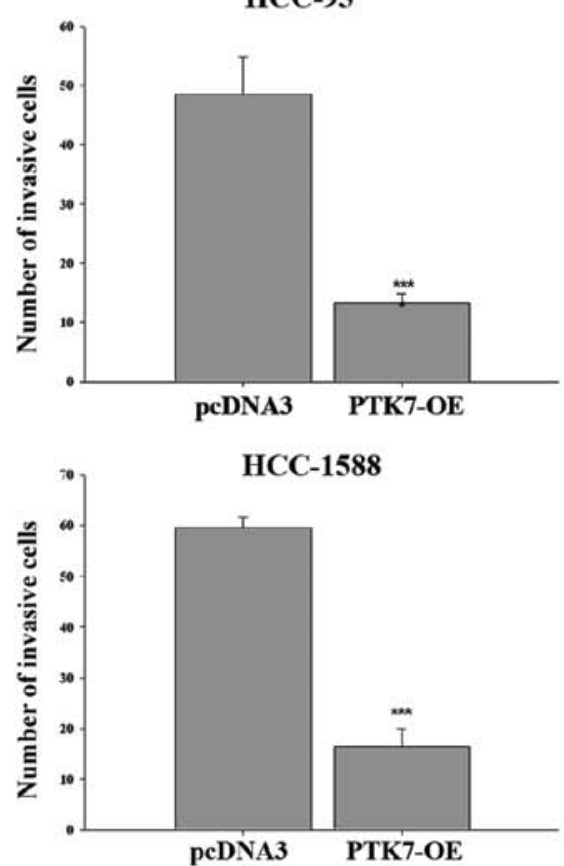

Figure 3. Overexpression of PTK7 inhibits proliferation, wound healing and invasion in LSCC cell lines. (A) HCC-95 and HCC-1588 cells were transfected with empty vector (pcDNA3) or pcDNA3-PTK7-flag and protein expression was confirmed by western blotting. (B) Effect of PTK7 overexpression (PTK7-OE) on proliferation in the HCC-95 (left panel) and HCC-1588 (right panel) cells. (C) Effect of PTK7-OE on wound healing in HCC-95 and HCC-1588 LSCC cells (left). The number of migrated cells (right). Light micrographs (x100) showing wound healing in monolayers of HCC-95 and HCC-1588 cells with or without PTK7-OE. (D) Effect of PTK7-OE on Matrigel invasion of HCC-95 and HCC-1588 LSCC cells. ${ }^{*} \mathrm{P}<0.05,{ }^{* * * *} \mathrm{P}<0.001$ vs. control (pcDNA3).

regulated, depending on the organ and tumor and that disease prognosis would differ according to the rate of PTK7 expression. However, expression of PTK7 and its role as a tumor suppressor in LSCC have not been studied.
In the current study, we showed that PTK7 expression is downregulated by approximately $>80 \%$ in tumor cells from tissue samples from LSCC patients in comparison to that observed in normal tissues (control) at both mRNA and 
$\mathbf{A}$

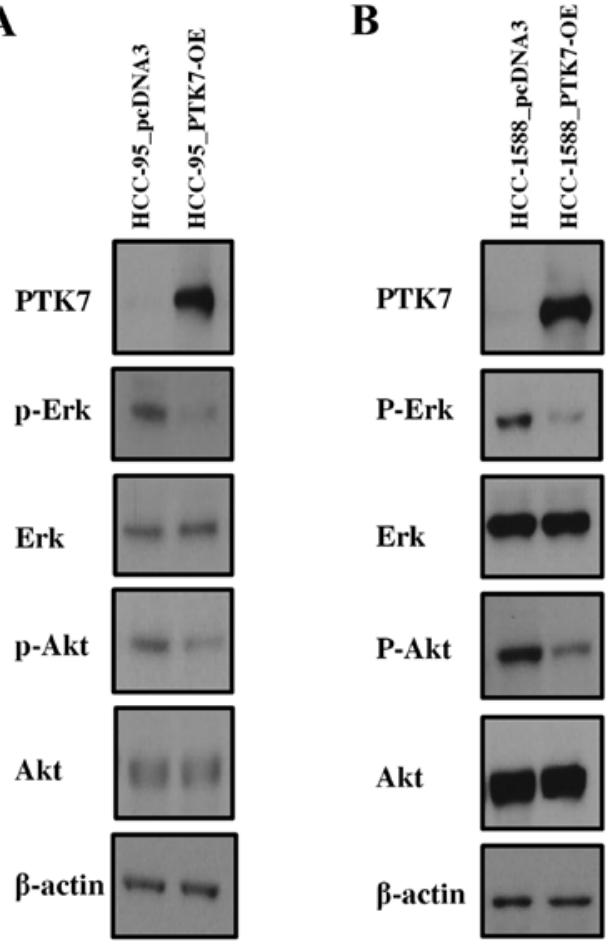

Figure 4. Effects of PTK7 overexpression on the activation of signaling proteins in HCC-95 and HCC-1588 LSCC cells. Expression of signaling proteins ERK and AKT was assessed by western blotting analysis in (A) HCC-95 and (B) HCC-1588 cells with vector or PTK7-OE. $\beta$-actin levels are shown for normalization

protein levels. PTK7 expression was also low at both levels in the LSCC cell lines. Based on these results, we sought to link PTK7 expression to tumor suppression in LSCC. In the present study, we found that PTK7 overexpression decreases the proliferation and inhibits wound healing and invasion of HCC-95 and HCC-1588 LSCC cells, although no significant difference in growth was observed between control and PTK7-overexpressing cells. Moreover, we found that overexpression of PTK7 decreased the phosphorylation of AKT and ERK in LSCC cells. AKT and MAPK are important signaling cascades involved in cell proliferation, tumor invasion and migration (17-20). Therefore, we suggest that PTK7 may be involved in tumor and metastasis repression in LSCC, particularly through inactivation of AKT and MAPK, which promote proliferation, invasion and migration.

In conclusion, our results show that PTK7 is downregulated in clinical samples of LSCC and that it plays a tumor suppressor role (via the AKT and MAPK pathways) in LSCC cells. However, further studies are required to elucidate the tissue and organ-specific differences in the oncogenic patterns of PTK7 and to determine their clinical significance in LSCC.

\section{Acknowledgements}

This work was supported by grants from the National R\&D Program for Cancer Control (1120260 to J.H.P), Ministry of Health and Welfare of the Republic of Korea, and from the Radiological Translational Research Program (RTR), Korea Institute of Radiological \& Medical Sciences (KIRAMS 50455-2013).

\section{References}

1. Parkin DM, Bray F, Ferlay J and Pisani P: Global cancer statistics, 2002. CA Cancer J Clin 55: 74-108, 2005.

2. Koyi H, Hillerdal G and Brandèn E: A prospective study of a total material of lung cancer from a county in Sweden 1997-1999: gender, symptoms, type, stage, and smoking habits. Lung Cancer 36: 9-14, 2002.

3. Visbal AL, Williams BA, Nichols FC,3rd, Marks RS, Jett JR, Aubry MC, Edell ES, Wampfler JA, Molina JR and Yang P: Gender differences in non-small-cell lung cancer survival: an analysis of 4,618 patients diagnosed between 1997 and 2002. Ann Thorac Surg 78: 209-215, 2004.

4. Mossie K, Jallal B, Alves F, Sures I, Plowman GD and Ullrich A: Colon carcinoma kinase- 4 defines a new subclass of the receptor tyrosine kinase family. Oncogene 11: 2179-2184, 1995.

5. Lin Y, Zhang L, Wang X, Xing X, Cheng X, Dong B, Hu Y, Du H, Li Y and Zhu Y: PTK7 as a novel marker for favorable gastric cancer patient survival. J Surg Oncol 106: 880-886, 2012.

6. Shin WS, Kwon JH, Lee HW, Kang MC, Na HW, Lee ST and Park JH: The oncogenic role of PTK7 in esophageal squamous cell carcinoma. Cancer Sci 104: 1120-1126, 2013.

7. Gobble RM, Qin L, Brill ER, Angeles CV, Ugras S, O'Connor RB, Moraco NH, DeCarolis PL, Antonescu C and Singer S: Expression profiling of liposarcoma yields a multigene predictor of patient outcome and identifies genes that contribute to liposarcomagenesis. Cancer Res 71: 2697-2705, 2011.

8. Meng L, Sefah K, O'Donoghue MB, Zhu G, Shangguan D, Noorali A, Chen Y, Zhou L and Tan W: Silencing of PTK7 in colon cancer cells: caspase-10-dependent apoptosis via mitochondrial pathway. PLoS One 5: e14018, 2010.

9. Behbahani TE, Thierse C, Baumann C, Holl D, Bastian PJ, von Ruecker A, Müller SC, Ellinger J and Hauser S: Tyrosine kinase expression profile in clear cell renal cell carcinoma. World J Urol 30: 559-565, 2012.

10. Easty DJ, Mitchell PJ, Patel K, Florenes VA, Spritz RA and Bennett DC: Loss of expression of receptor tyrosine kinase family genes PTK7 and SEK in metastatic melanoma. World J Urol 71: 1061-1065, 1997.

11. Su YA, Yang J, Tao L, Nguyen H and He P: Undetectable and decreased expression of KIAA1949 (Phostensin) encoded on chromosome $6 \mathrm{p} 21.33$ in human breast cancers revealed by transcriptome analysis. J Cancer 38: 38-50, 2010.

12. Na HW, Shin WS, Ludwig A and Lee ST: The cytosolic domain of protein-tyrosine kinase 7 (PTK7), generated from sequential cleavage by a disintegrin and metalloprotease 17 (ADAM17) and $\gamma$-secretase, enhances cell proliferation and migration in colon cancer cells. J Biol Chem 287: 25001-25009, 2012.

13. Shin WS, Maeng YS, Jung JW, Min JK, Kwon YG and Lee ST: Soluble PTK7 inhibits tube formation, migration, and invasion of endothelial cells and angiogenesis. Biochem Biophys Res Commun 371: 793-798, 2008.

14. Lee SJ, Yoo HJ, Bae YS, Kim HJ and Lee ST: TIMP-1 inhibits apoptosis in breast carcinoma cells via a pathway involving pertussis toxin-sensitive $\mathrm{G}$ protein and $\mathrm{c}-\mathrm{Src}$. Biochem Biophys Res Commun 312: 1196-1201, 2003.

15. Endoh H, Tomida S, Yatabe Y, Konishi H, Osada H, Tajima K, Kuwano H, Takahashi T and Mitsudomi T: Prognostic model of pulmonary adenocarcinoma by expression profiling of eight genes as determined by quantitative real-time reverse transcriptase polymerase chain reaction. J Clin Oncol 22: 811-819, 2004.

16. Garber ME, Troyanskaya OG, Schluens K, Petersen S, Thaesler Z, Pacyna-Gengelbach M, van de Rijn M, Rosen GD, Perou CM and Whyte RI: Diversity of gene expression in adenocarcinoma of the lung. Proc Natl Acad Sci USA 98: 13784-13789, 2001.

17. Manning BD and Cantley LC: AKT/PKB signaling: navigating downstream. Cell 129: 1261-1274, 2007.

18. Johnson GL and Lapadat R: Mitogen-activated protein kinase pathways mediated by ERK, JNK, and p38 protein kinases. Science 298: 1911-1912, 2002.

19. Shin I, Kim S, Song H, Kim HR and Moon A: H-Ras-specific activation of Rac-MKK3/6-p38 pathway: its critical role in invasion and migration of breast epithelial cells. J Biol Chem 280: 14675-14683, 2005.

20. Huang C, Jacobson K and Schaller MD: MAP kinases and cell migration. J Cell Sci 117: 4619-4628, 2004. 